\title{
In Silico Analysis of Synaptonemal Complex Protein 1 (SYCP1) and Acrosin Binding Protein (ACRBP) Antigens to Design Novel Multiepitope Peptide Cancer Vaccine Against Breast Cancer
}

\author{
Ashkan Safavi ${ }^{1} \cdot$ Amirhosein Kefayat $^{2} \cdot$ Fattah Sotoodehnejadnematalahi $^{1} \cdot$ Mansoor Salehi $^{3}$. \\ Mohammad Hossein Modarressi ${ }^{1}$
}

Accepted: 15 October 2018 / Published online: 25 October 2018

(c) Springer Nature B.V. 2018

\begin{abstract}
Multiepitope cancer vaccines are manifesting as the future of breast cancer immunotherapy. In the present study, high immunogenic fragments of synaptonemal complex protein 1 (SYCP1) and acrosin binding protein (ACRBP) antigens were selected according to MHCs binding affinity and $\mathrm{CD} 8^{+}$cytotoxic T lymphocytes' (CTL) epitopes by various immunoinformatic servers. (RCQHKIAEMVALMEKHKHQYDKI) residue from p702-724 SYCP1 and the (YIQYPNYCSFKSQQCL) residue from p481-496 ACRBP were selected as the immunodominant fragments. Then, the selected fragments were fused together with a furin-sensitive linker to form the final peptide vaccine construct. The cleavage sites, TAP transport efficiency, CTL epitopes, post-translational modifications, and B cells epitopes were predicted for the final construct. The final construct contained appropriate CTLs epitopes and also, several linear and conformational B cell epitopes. Also, it exhibited 90.37\% HLA population coverage in the world. Therefore, these preliminary results require validation by in vitro and in vivo experiments.
\end{abstract}

Keywords Immunoinformatic $\cdot$ Cancer/testis antigen $\cdot$ SYCP1 $\cdot$ ACRBP $\cdot$ Multiepitope peptide vaccine $\cdot$ Breast cancer

Abbreviations
$\begin{array}{ll}\text { SYCP1 } & \text { Synaptonemal complex protein } 1 \\ \text { ACRBP } & \text { Acrosin binding protein } \\ \text { TAAs } & \text { Tumor-associated antigens } \\ \text { CTAs } & \text { Cancer/testis antigens } \\ \text { MHC } & \text { Major histocompatibility complex } \\ \text { NCBI } & \text { National Center for Biotechnology Information } \\ \text { CAI } & \text { Codon Adaptation Index } \\ \text { CTL } & \text { Cytotoxic T cell lymphocyte } \\ \text { HLA } & \text { Human leukocytes antigen }\end{array}$

Electronic supplementary material The online version of this article (https://doi.org/10.1007/s10989-018-9780-z) contains supplementary material, which is available to authorized users.

Mohammad Hossein Modarressi

modaresi@tums.ac.ir

1 Department of Biology, Science and Research Branch, Islamic Azad University, Tehran, Iran

2 Department of Oncology, Cancer Prevention Research Center, Isfahan University of Medical Sciences, Isfahan, Iran

3 Division of Genetics and Molecular Biology, School of Medicine, Isfahan University of Medical Sciences, Isfahan, Iran
TAP Transporter associated antigen presentation

PTM Post-translational modifications

\section{Introduction}

Breast cancer is the leading cause of cancer death among women (Khanfir et al. 2013). Although breast cancer treatments have been considerably improved in recent years, patients' prognosis, especially for metastatic breast cancer, is not satisfying (Arpino et al. 2015; Redig and McAllister 2013). Tumors arise from a combination of genetic and epigenetic changes which lead to new cellular characterizations like immortality and uncontrolled proliferation (Erenpreisa and Cragg 2013; Ghahremani et al. 2018). These transformations cause new antigens expression in malignant cells. In addition, the immune system has the ability to recognize these changes and eliminate the cancer cells (Schumacher and Schreiber 2015). However, some cancer cells get the chance to escape the immune system's recognition (Whiteside et al. 2011; Beatty and Gladney 2015). Therefore, appropriate antigen selection and presentation to the immune system can effectively stimulate the immune response (Shastri et al. 2002). 
Vaccination is an effective strategy for introducing specific tumor antigens to the immune system. Many different types of vaccines including peptide and DNA vaccines have been utilized for breast cancer treatment (Vinay et al. 2015). DNA vaccines have exhibited appropriate stability, simplicity, and ability to introduce several antigens and epitopes to the immune system (Yang et al. 2014; Tiptiri-Kourpeti et al. 2016). Clinical trials of DNA vaccines against HER2 (Norell et al. 2010) and mammaglobin (Tiriveedhi et al. 2013) have shown promise in stimulating immune responses against breast tumor antigens (Yang et al. 2014). Also, peptide vaccines have gained lots of attention for breast cancer treatment. Vaccines containing derivative epitopes of breast tumor antigens such as mucin 1, human epidermal growth factor receptor 2 (HER2/neu), and carcinoembryonic antigen (CEA) are under investigation for use against breast cancer. They have been shown to provide immunogenic value without causing autoimmunity in phase I/II/III clinical trials (de Peres 2015; Thundimadathil 2012; Finn 2003). Although the peptide vaccine therapy shows encouraging responses, there are still challenges with respect to the variety of evasion mechanisms of tumor cells from the immune system (de Peres 2015; Karkada 2013). Therefore, there is a need to improve the immunogenicity of peptide cancer vaccines.

Appropriate selections of antigens for cancer immunization can profoundly affect the cancer vaccine efficacy. Tumor-associated antigens (TAAs) are the main utilized antigens for designing cancer vaccines (Vigneron 2015). TAAs are consisted of a vast variety of antigens including differentiation antigens (e.g. Melan-A/MART-1) (Coulie et al. 1994), mutational antigens (e.g. CTNNB1) (Robbins et al. 1996), overexpressed antigens (e.g. HER2) (Kraus et al. 1987), viral antigens (e.g. human papillomavirus proteins) (Demetris Iacovides et al. 2013), and cancer/testis antigens (CTAs) (Lurquin et al. 1997; Chomez et al. 2001). CTAs (e.g. SYCP1, ACRBP, NY-ESO-1, SSX2, etc.) are one of the most well-known TAAs. They are named after their restricted expression in testicular and cancerous tissues (Tarnowski 2016). Therefore, CTAs can be appropriate choices for anti-cancer immunization according to many cancer vaccines clinical trials (Zajac et al. 2017; Gjerstorff et al. 2015).

SYCP1 and ACRBP are members of CTAs families in which the spontaneous immune responses against both of them have been detected in cancer patients (TaherianEsfahani 2016). SYCP1 interacts with different proteins as a part of the synaptonemal complexes during meiosis and ACRBP participates in spermatogenesis and sperm capacitation (Whitehurst et al. 2010; Costa et al. 2005; Nielsen and Gjerstorff 2016). ACRBP and SYCP1 are located at 12 p12-p13 and 1p13.2, respectively (Ono 2001; Xia et al. 2014; Türeci 1998). ACRBP expression has been detected in various types of cancer including bladder (Ono 2001), ovarian (Tammela et al. 2006), and colorectal cancer (Ono 2001).
Also, SYCP1 is expressed at brain (Türeci 1998; Sahin et al. 2000), pancreas (Kubuschok et al. 2004), and hepatobiliary cancers (Luo et al. 2002). Mashino et al. detected high expression of SCYP1 at about $34 \%$ of breast cancer patients' tumors (Mashino et al. 2001). Tureci et al. have observed anti-SYCP1 serum reactivity in half of the breast cancer patients (Türeci 1998). Also, many studies have reported high expression of ACRBP in breast tumors (Ono 2001; Whitehurst 2010a, b). Therefore, it seems SYCP1 and ACRBP can be ideal antigens for designing breast cancer vaccine.

The epitope-based vaccines have many advantages over whole-protein antigens immunization, such as enhanced safety, high immunogenicity, and protective potency (Beatty and Gladney 2015; Tang et al. 2015; Gu et al. 2017). Therefore, immunodominant epitopes have long been used to design anti-cancer peptide vaccine (Ebert et al. 2009; Iinuma et al. 2014). Experimental methods for determining the immunodominant epitopes are time-consuming, expensive, and limited. Thus, employing new computational methods for identifying immunodominant epitopes has received lots of attention (Mahdavi et al. 2012; Chen et al. 2011).

Immunological bioinformatics (immunoinformatic) methods are trustable tools for these purposes. They focus on the structure, function, and interactions of the molecules involved in immunity. Many immunoinformatic servers and databases have been introduced for the better understanding of the immune system and its probable responses (SoriaGuerra et al. 2015). Their epitope mapping can identify the $\mathrm{T}$ cell epitopes which are determinative in the anti-cancer immune response. This fact has significantly enhanced antigens immunodominant epitopes determination, multiepitope vaccines designing and predicting their immunogenicity and binding affinity for the major histocompatibility complex (MHC) molecules (Fratta et al. 2011; Patronov and Doytchinova 2013). Hence, employing immunoinformatic tools for designing cancer vaccine could be more efficient.

In the present study, immunoinformatic tools were employed for designing novel multiepitope peptide and DNA cancer vaccine consisted of immunodominant fragments of SYCP1 and ACRBP antigens for breast cancer immune therapy.

\section{Methods}

\section{Study Design}

First, the similar sequences between human and mouse SYCP-1 and ACRBP antigens were identified. The most appropriate regions were selected from the similar candidate sequences according to containing immunodominant epitopes by bioinformatics tools. Subsequently, selected 
fragments were linked together by an furin-sensitive linker and the final construct was analyzed at different aspects by different servers. The final construct was analyzed at different aspects by different servers. Reverse translation of the final construct to DNA and adaptation of the DNA sequence to Escherichia coli and mouse codon usage were done by bioinformatics servers. A flowchart model was designed to represent the step by step workflow of the study (Fig. 1).

\section{Homology Analysis to Identify Candidate Regions}

The SYCP1 and ACRBP amino acid sequences were obtained from NCBI (http://www.ncbi.nlm.nih.gov). The similar regions between human and mouse (100\% homology) were recognized by Protein Blast (https://blast.ncbi. nlm.nih.gov/Blast.cgi). Then, all the regions were compared with both mouse and human proteome to recognize the regions without any similarity with both species' proteome (unique regions) for using in the next sections.

\section{Immunoinformatic Analysis}

\section{Prediction of MHC I and II Binding Peptides Affinity}

Artificial neural network (ANN) and stabilized matrix method (SMM) methods in "IEDB" server (http://tools .immuneepitope.org/analyze/html/mhc_binding.html) (Nezafat et al. 2014) that used $\mathrm{IC}_{50}[\mathrm{nM}]<50$ as the cut off and NetMHCpan 4.0 (http://www.cbs.dtu.dk/services/ NetMHCpan/) (Vita et al. 2009) were employed to predict MHC-I binding affinity for both human and mouse alleles The percentile rank $<1 \%$ was used. In addition, ProPred-I

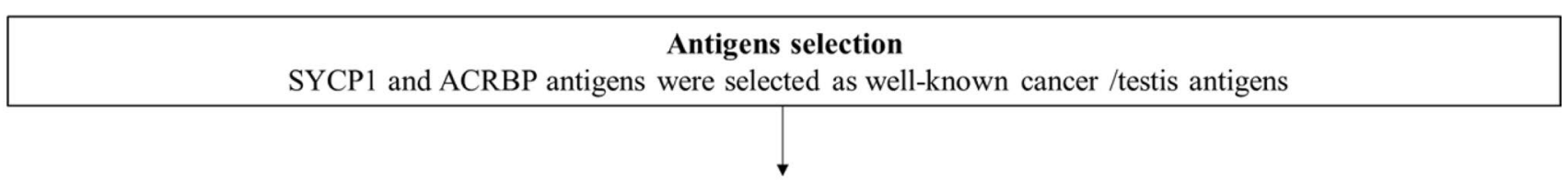

Identification of the candidate regions

Alignment of mouse and human antigens amino acids sequences and identification of the homologous regions $\downarrow$

\section{Selection of immunodominant region of each antigen}

Prediction of the CTL epitopes and MHCs binding affinity of each candidate regions and selection of the most immunodominant regions of SYCP1 and ACRBP

\section{Designing the multiepitope peptide vaccine final construction}

Fusing the two selected immunodominant regions by a furin sensitive linker and final refinements and modifications

\begin{tabular}{l} 
Predicting the 3D structure of the multiepitope peptide vaccine \\
Prediction, refinement, and validation of 3D structure of the peptide vaccine by several serves \\
Prediction of the conformational and linear B cell epitopes according to the 3D structure \\
Predicting B cell epitope of the multiepitope peptide vaccine \\
Revers translation of the multiepitope peptide vaccine, codon optimization, and adding suitable restriction enzyme sites \\
\hline
\end{tabular}

Fig. 1 The flow chart of the multiepitope peptide and DNA vaccines designing 
(http://www.imtech.res.in/raghava/propred1/) (Singh and Raghava 2003) was employed for confirming the MHC-I binding ability. The maximum accuracy of ProPred-I is $75 \%$ at $4 \%$ threshold (default threshold). SMM-align, and $\mathrm{NN}$-align, methods in "IEDB" (http://tools.immuneepitope. org/analyze/html/mhc_binding.html) and MHCpred (http:// www.ddg-pharmfac.net/mhcpred/MHCPred/) (Guan et al. 2003) was employed to recognize MHC-II binding peptides. The default cut-off value of $\mathrm{IC}_{50}$ was used. Also, NetMHCIIpan 3.2 (http://www.cbs.dtu.dk/services/ NetMHCIIpan/) (Jensen 2018) server was used and the threshold for strong binder was set at (\% Rank) $\leq 2$. The used reference set of MHC I and II alleles had more than $97 \%$ and $99 \%$ population coverage in all major ethnicities worldwide, respectively (Greenbaum et al. 2011; Weiskopf 2013; Khalili et al. 2015).

\section{CD8 ${ }^{+}$Cytotoxic T Lymphocytes (CTL) Epitopes Prediction}

Three CTL prediction tools including NetCTLpan 1.1 (http://www.cbs.dtu.dk/services/NetCTLpan/) (Khalili et al. 2015; Stranzl et al. 2010) with experimental verifications of $>96 \%$ to identify new epitopes with $90 \%$ likelihood, NetCTL 1.2 (http://www.cbs.dtu.dk/services/NetCTL/) (Pandey et al. 2018; Larsen et al. 2007) using all the server provided optional HLA supertypes (threshold for epitope identification $>0.75$ ) and both ANN and SVM prediction approaches of CTLPred (http://www.imtech.res.in/raghava/ ctlpred/) (Khalili et al. 2015; Bhasin and Raghava 2004). The NetCTLpan 1.1 method integrates prediction of peptide MHC class I binding, proteasomal C terminal cleavage, and TAP transport efficiency. MHC class I binding and proteasomal cleavage is performed using artificial neural networks. TAP transport efficiency is predicted using weight matrix. For NetCTLpan 1.1 percentile Rank $<1 \%$ was approved.

\section{Peptide Final Construct}

The selected fragments of the antigens were linked together by a furin-sensitive amino acid linker (RVRR). A methionine and a glycine were added to the $\mathrm{N}$-terminal. In addition, His-tag (six histamines) was added to the C-terminal to form the final peptide construct.

\section{Vaccine Features}

\section{Prediction of Protein Solubility and Stability}

To predict the protein solubility properties, SOLpro (http:// scratch.proteomics.ics.uci.edu/) (Magnan et al. 2009) which utilizes a two-stage SVM architecture method based on multiple representations of the primary sequence was used. The overall accuracy of SOLpro is estimated over $74 \%$ using multiple runs of tenfold cross-validation. In addition, various physicochemical parameters including amino acid composition, theoretical $\mathrm{pI}$, instability index, in vitro and in vivo half-life, aliphatic index, grand average of hydropathicity (GRAVY), and molecular weight were evaluated by ProtParam online server (http://web.expasy.org/protparam/) (Gasteiger et al. 2005).

\section{Proteasomal Cleavage and TAP Transport Prediction}

NetChop (http://tools.iedb.org/netchop/) and MAPPP (http:// www.mpiib-berlin.mpg.de/MAPPP/ cleavage.html) (Subramanian and Chinnappan 2013) were employed for predicting the proteasomal processing. In addition, TAPPred (http:// crdd.osdd.net/raghava/tappred/index.html) (Sankar et al. 2017) was used to evaluate the binding affinity of the peptides with the TAP transporter.

\section{Tertiary Structure}

\section{Homology Modeling and De Novo Structure Prediction}

The final construct complete 3D structure was determined by employing Phyer2 (http://www.sbg.bio.ic.ac.uk/phyre 2/html/page.cgi?id=index) and SWISS-MODEL (https:// swissmodel.expasy.org/interactive) (Kelley and Sternberg 2009; Guex et al. 2009) which utilize alignment of the target sequence and template structures for homology modeling. For de novo prediction of the 3D structure according to the amino acid sequence, PEP-FOLD (http://bioserv.rpbs.univparis-diderot.fr/services/PEP-FOLD/) and CABS (http:// biocomp.chem.uw.edu.pl/CABSfold/) (Maupetit et al. 2009; Blaszczyk et al. 2013) were employed. These servers need the secondary structure for refinement of the tertiary structure of the protein. Therefore, PSIPRED V3.3 (http://bioin f.cs.ucl.ac.uk/psipredtest) (EL-Manzalawy et al. 2008) was employed for the prediction of the secondary structure.

\section{Tertiary Structure Refinement}

The obtained 3D structure was processed by GalaxyLoop server (http://galaxy.seoklab.org/) for generating loop modeling. The loop refining was accomplished by specifying the locations of loop regions in a protein model with ab initio modeling method (Shin et al. 2014; Ko et al. 2012). In the next step, more refinements were done by ReFOLD (http:// www.reading.ac.uk/bioinf/ReFOLD/) (Shuid et al. 2017; McGuffin et al. 2017), ModRefiner (https://zhanglab.ccmb. med.umich.edu/ModRefiner/) and Galaxyrefine (http://galax y.seoklab.org/) (Xu and Zhang 2011) to have the most optimized model. 


\section{Tertiary Structure Validation}

In order to find the potential errors in initial 3D models, ProSA-web (https://prosa.services.came.sbg.ac.at/ prosa.php) (Wiederstein and Sippl 2007), PROCHECK program (http://swissmodel.expasy.org/workspace/index .php?func $=$ tools_structureassessment1) (Laskowski et al. 1993) and ERRAT server (http://nihserver.mbi.ucla.edu/ ERRATv2/) (Colovos and Yeates 1993) were utilized. ProSA-web calculates the overall quality score by analyzing atomic coordination of the model. The ProSA-web $\mathrm{z}$-score is indicated in a plot, which contains the $\mathrm{z}$-score of experimentally determined structures deposited in PDB. The residue-by-residue stereochemical qualities of models were validated by Ramachandran plot obtained from PROCHECK. The ERRAT program evaluates the statistics of non-bonded atom-atom interactions in compared to a database of reliable high-resolution crystallography structures (Kozakov 2010).

\section{B Cell Epitopes Prediction}

In order to recognize linear and conformational B cell epitopes, BCPREDS (http://ailab.ist.psu.edu/bcpred/predi ct.html) was employed. BCPred method employs subsequence kernel-based SVM classifier and was trained on homology-reduced dataset of linear B-cell epitopes (with $<80 \%$ sequence identity) derived from dataset previously used to test ABCPred. ElliPro (http://tools.iedb.org/ellipro/) (Ponomarenko et al. 2008) and DiscoTope (http://www.cbs. dtu.dk/services/DiscoTope/) (Kringelum et al. 2012) servers were utilized. Conformational discontinuous B-Cell epitopes were computationally determined by ElliPro tool at IEDB with 0.7 as a minimum Protrusion Index and $6 \AA$ as a maximum distance. The ElliPro tool allowed identification of B-Cell epitopes in a submitted protein three-dimensional structure as a PDB file after homology modeling. ElliPro runs on a database of discontinuous determinants produced from three-dimensional structures of antigen-antibody complexes have shown to have highest accuracy in comparison to six adjacent tools (Ponomarenko et al. 2008). For the DiscoTope serve the default threshold $(-3.7)$ was used for identification of epitopes. In this threshold, the sensitivity and specificity are 0.47 and 0.75 , respectively.

\section{Population Coverage}

To find out the population coverage of each fragment and the final construct, Epitope/MHC restriction data were loaded at the Population Coverage Calculation tool of IEDB (http://tools .immuneepitope.org/tools/population/iedb_input) (Khalili et al. 2015). Calculations were set to be performed for MHC class I and II epitopes.

\section{Post-translational Modifications}

Post-translational modifications of the DNA vaccine translated peptide including O-glycosylation (http://www.cbs. dtu.dk/services/NetOGlyc/) (Steentoft et al. 2013), N-glycosylation (http://www.cbs.dtu.dk/services/NetNGlyc/) (Blom et al. 2004), phosphorylation (http://www.cbs.dtu.dk/servi ces/NetPhos/) (Blom et al. 1999), and ubiquitination (http:// www.ubpred.org/) (Radivojac 2010) were predicted. The existence of PEST motifs was analyzed by using PEST-find server (Khalili et al. 2015; Moradi 2017). Protein functional family prediction was performed for the final construct by SVMProt network service based on SVM classification (Khalili et al. 2015).

\section{Data Validation}

To evaluate the proper cleavage probability of the proposed epitopes and their availability to the host immune system, retrieval of the original CTL epitopes in the context of final construct was predicted using CTLPred, NetCTL 1.2 and NetCTLpan 1.1, EpiJen, (http://www.ddgpharmfac.net/ epije n/EpiJen/EpiJen.htm) (Doytchinova et al. 2006), RANKPEP (http://imed.med.ucm.es/Tools/rankpep.html) (Reche et al. 2004) and SYFPEITHI (http://www.syfpeithi.de/) (Rammensee et al. 1999). The main processing pathway for MHC class I ligands involves degradation of proteins by the proteasome, followed by transport of products by the transporter associated with antigen processing (TAP) to the endoplasmic reticulum (ER) where peptides are bound by MHC class I molecules and then presented on the cell surface by MHCs. The whole process is modeled using EpiJen. EpiJen is based on quantitative matrices, derived by the additive method and applied successively to select epitopes. In this study, 5\% threshold was selected, giving $85 \%$ sensitivity (Doytchinova et al. 2006). SYFPEITHI contains more than 7000 peptide sequences of human and other organisms, such as chicken, mouse, and apes, which are known to bind MHC-I and MHC-II molecules (Rammensee et al. 1999). In addition, the final construct population coverage was reinvestigated by the used server at "Vaccine Features" section.

\section{Multiepitope DNA Construct}

The template DNA sequence was reverse translated from the peptide sequence and codon optimized by the JCAT (http://www.jcat.de/). The JCAT presents a simple method to adapt the Codon Usage to the most of sequenced prokaryotic organisms and selected eukaryotic organisms. The CAIvalues were calculated by applying an algorithm from A. 
Carbone et al. For more analyzes, GenScript (https://www. genscript.com/tools/rare-codon-analysis) was utilized which can calculate GC content and codon frequency distribution (CFD). The CFD is another factor that can affect expression efficiency. (CAI) $>0.8$ was selected to support mouse codon adaptation. The GC content among 30-70\% was accepted for high-level expression at the desired host. In addition, the Kozak sequence was added to the 5' of the nucleotide sequences to enhance its expression. At last, the DNA construct with suitable restriction enzyme sites (Nhe1, Nco1, and Xho1) at the 5' and 3' ends was designed.

\section{Results}

\section{Identification of the Candidate Regions}

To design an effective vaccine for both human and mouse due to the necessity of pre-clinical animal experiments before planning human clinical trials, the human and murine amino acid sequences were aligned and only the similar regions (100\% homology) were selected (Figs. 2, 3). This will avoid selecting of regions with different sequences between the two species. The similar regions with appropriate length were identified and listed in Tables $\mathrm{S} 1$ and S2. Peptides' appropriate length for MHC-I and MHC-II binding is 8-9 and 12-15 amino acids, respectively. One of the ACRBP regions had similar sequences with some other proteins in the proteome and subsequently was omitted to prevent tolerances. Therefore, just the similar regions with unique sequences in proteome were selected as candidate regions for immunoinformatic analysis (Tables S1, S2).

\section{Selection of the Most Immunogenic Regions of Antigens}

The (RCQHKIAEMVALMEKHKHQYDKI) region from SYCP1 isoform 1 and (YIQYPNYCSFKSQQCL) region from ACRBP were selected as the most immunogenic regions. These regions contained multiple epitopes with high affinity for binding to several MHCs. Their epitopes with the high binding affinity $\left(\mathrm{IC}_{50}\right.$ values $<50 \mathrm{nM}$ or confirmed with ProPred-I) are listed in Tables S3 and S4. The average world population coverage of the SYCP1 and ACRBP selected fragments were about $80 \%$ and $71 \%$, respectively (Table S5). CTL epitopes are so important

\begin{tabular}{|c|c|c|c|}
\hline Human & 1 & AAGFLPSLLKVLLLPLAPAAAQDSTQASTPGS PLSPTEYERFFALLTPTWKAETTC & 60 \\
\hline & & P +A++S ASTPGSPLS TEYERFFALLTPTWKAETTC & \\
\hline ouse & 1 & MLLEVLLLPGTPLSAEESP-ASTPGS PLSSTEYERFFALLTPTWKAETTC & \\
\hline Iuman & 61 & RLRATHGCRNPTLVQLDQYENHGLVPDGAVCSNL PYASWFES FCQFTHYRCSNHVYYAKR & \\
\hline & & RLRATHGCRNPTLVQLDQYENHGLVPDGAVCS+LPYASWFESFCQF & \\
\hline ouse & 60 & RLRATHGCRNPTLVQLDQYENHGLVPDGAVCSDL PYASWFES FCQFAQYRCSNHVYYAKR & \\
\hline Human & 121 & $\begin{array}{l}\text { VLCSQPVSILSPNTLKE IEASAEVSPTTMTSPISPHFTVTERQTFQPWPERLSNNVEELL } \\
\text { CSOPVSILSPNTLKE+E+SAEV PT+MT+PI H T TE O FOPWPERL+NNVEELL }\end{array}$ & \\
\hline e & 20 & LRCSQPVSILS PNTLKEVESSAEVPPTSMTTPIVSHATATEHQAFQPWPERLNNNVEELL & \\
\hline Human & 181 & $\begin{array}{l}\text { QSSLSLGGQEQAPEHKQEQGVEHRQE PTQEHK-QEEGQKQEEQEEEQEEEGKQEEGQGTK } \\
\text { QSSLSLGG }++Q \quad+Q \quad+\mathrm{Q}+Q \mathrm{E} \quad \mathrm{QEHK} \quad+\mathrm{E} \quad+++\mathrm{E}++\mathrm{EEE}+\mathrm{EEE} \quad \mathrm{KQEEGQGT}+\end{array}$ & \\
\hline se & 80 & QSSLSLGGKDQQSSRRPGQE-QHKQEQIQEHKLEEAQEQEEQEEEEEEEEAKQEEGQGTE & \\
\hline Human & 240 & $\begin{array}{l}\text { EGREAVSQLQTDSEPKFHSESLSSNPSSFAPRVREVESTPMIMENIQELIRSAQEIDEMN } \\
\text { G E+VS+LQ+DSEPKF S+SLSSNPS F PRVREVES P++M+NIQELIRSAQE+DEMN }\end{array}$ & \\
\hline ouse & 239 & AGLESVSRLQSDSEPKFQSQSLSSNPSF FT PRVREVESAPLMMKNIQELIRSAQEMDEMN & \\
\hline Human & 300 & $\begin{array}{l}\text { EIYDENSYWRNQNPGSLLQLPHTEALLVLCYSIVENTCIITPTAKAWKYMEEEILGFGKS } \\
\text { E+YD++ WR+Q+ GSL QLPH E L+VLCYSI+ENTC +TPTAKAW YMEEEILGFG S }\end{array}$ & \\
\hline ol & 99 & ELYDDS--WRSQSTGSLQQLPHMETLMVLCYS IMENTCTMTPTAKAWSYMEEEILGFGDS & \\
\hline Hun & 360 & $\begin{array}{l}\text { VCDSLGRRHMSTCALCDFCSLKLEQCHSEASLQRQQCDTSHKTPFVSPLLASQSLS IGNQ } \\
\text { VCD+LGRRH + C LC FCSLKLEQCHSEAS+ RQ+CD SHK PF+SPLL++QS+S GNQ }\end{array}$ & \\
\hline 101 & 57 & VCDNLGRRHTAACPLCAFCSLKLEQCHSEASVVRQKCDASHKIP FISPLLSAQS ISTGNQ & \\
\hline Hum & 420 & $\begin{array}{l}\text { VGSPESGRFYGLDLYGGLHMDFWCARLATKGCEDVRVSGWLQTEFLSFQDGDFPTKICDT } \\
\qquad \begin{array}{l}\text { P+ GRF GL++YGGL +FWC RLA KGCED RVS WL+ EFLSFQ+GDFPTKICDT }\end{array}\end{array}$ & \\
\hline e & 17 & ARIPDKGRFAGLEMYGGLSSE FWCNRLAMKGCEDDRVSNWLKAEFLSFQEGDFPTKICDT & \\
\hline Human & 480 & $\begin{array}{l}\text { DYIQYPNYCSFKSQQCLMRNRNRKVSRMRCLQNETYSALSPGKSEDVVLRWSQEFSTLTL } \\
+Y I Q Y P N Y C S F K S Q Q C L+R N+N R K+S R M R C+N E \text { Y LS KSE+V+LRWSQEFSTL + }\end{array}$ & \\
\hline & 77 & NYIQYPNYCSFKSQQCLLRNQNRKMSRMRCMLNERYNVLSLAKSEEVILRWSQEFSTLAI & \\
\hline Human & 540 & $\begin{array}{l}\text { GQFG } \\
\text { GQFG }\end{array}$ & \\
\hline Sus & & GQFG & \\
\hline
\end{tabular}

Fig. 2 Alignment of murine and human ACRBP antigens' amino acid sequences. The regions which has 100\% similarity between human and mouse (candidate regions) are highlighted 


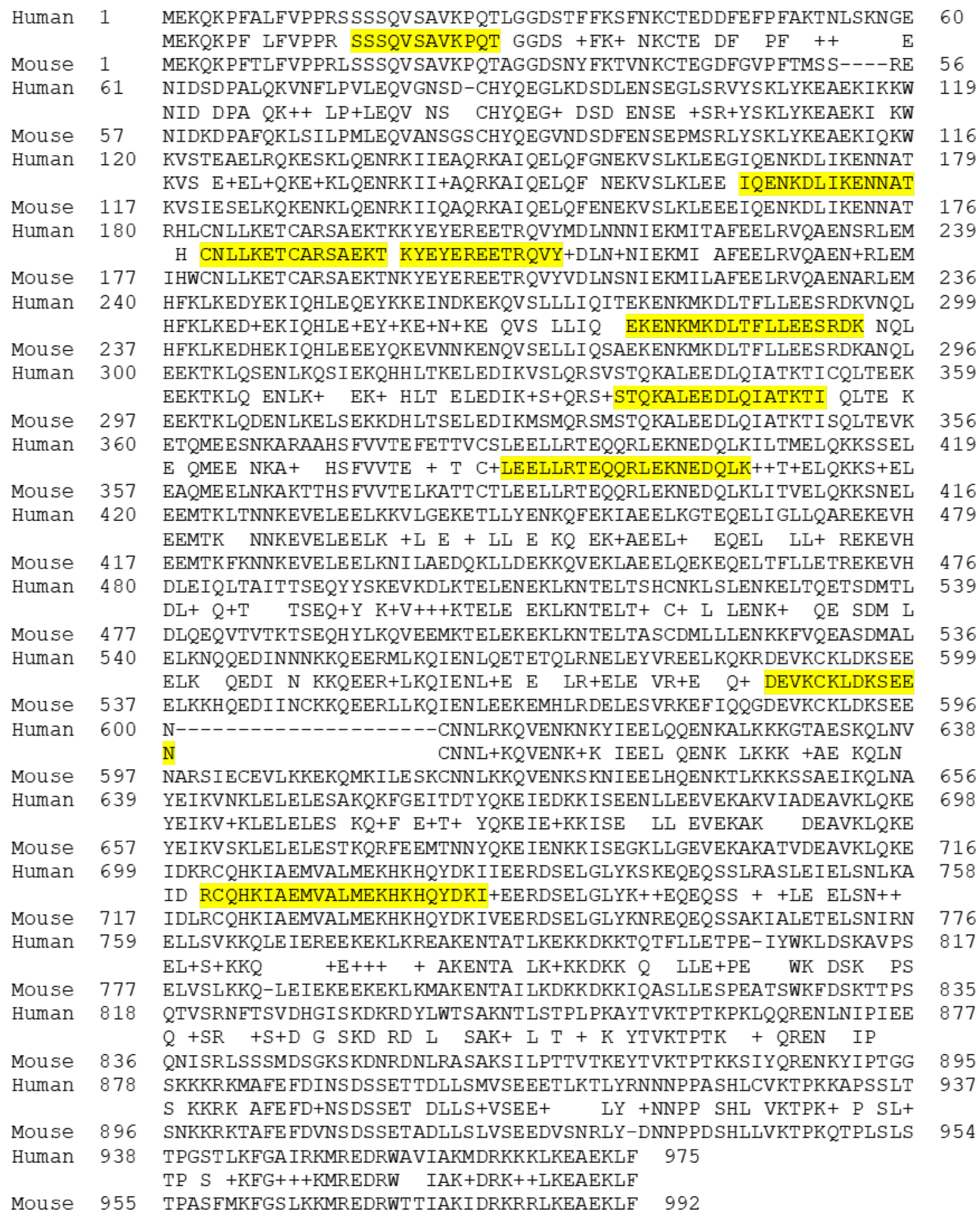

Fig. 3 Alignment of murine and human SYCP1 antigens' amino acid sequences. The candidate regions are highlighted

in cancer vaccine efficiency due to $\mathrm{T}$ lymphocytes critical role in the immune system anti-cancer response. Both fragments had multiple CTL epitopes which are listed in Tables S6 and S7.

\section{Final Construct of the Multiepitope Peptide Vaccine}

The final construct of the multiepitope peptide vaccine which had 51 amino acids length is schematically illustrated 


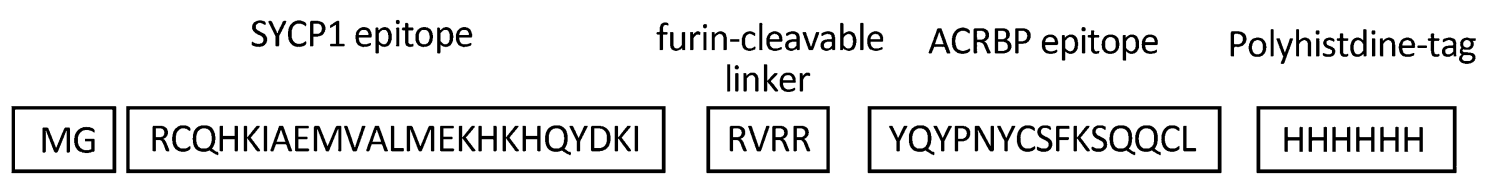

Fig. 4 Schematic diagram of the multiepitope vaccine amino acid sequence consists of methionine, glycine, SYCP1 region, furin-sensitive linker, ACRBP region, and His-tag

in Fig. 4. The construct is consisted of the two selected fragments which were fused to each other by the furin-sensitive linker. The methionine was added at the $\mathrm{N}$-terminal to form the AUG start code.

\section{The Multiepitope Peptide Vaccine Features}

\section{Solubility and Stability}

Peptides physio-chemical parameters have considerable effects on their properties like stability, transportation, and immunogenicity (Goodwin et al. 2012). The molecular weight $(\mathrm{Mw})$ for the multiepitope peptide vaccine was $6.38 \mathrm{kDa}$ and the theoretical isoelectric point value $(\mathrm{pI})$ was computed 9.61. In addition, the peptide solubility tendency upon overexpression in E. coli was 0.9 . Instability index predicts whether a protein is stable or unstable, if the predicted instability index of a protein is below 40 then it's called stable and proteins with the value above than 40 are comes under the category of unstable proteins. The instability index was computed 33.38 for the multiepitope peptide vaccine which categorizes as a stable protein. The estimated half-life in mammalian reticulocytes, yeast, and E. coli was about $30 \mathrm{~h}, 20 \mathrm{~h}$, and more than $10 \mathrm{~h}$, respectively. The grand average hydropathicity (GRAVY) and aliphatic indexes were defined -1.127 and 53.53, respectively. Negative value of GRAVY for the multiepitope peptide vaccine represents the hydrophilic nature of the peptide. It should be mentioned the peptides with positive value of GRAVY are hydrophobic. The estimated value of aliphatic index represents the thermostable nature of the multiepitope peptide vaccine and as the value of aliphatic index increases, the peptide exhibits greater thermo stability (Ikai 1980; Kyte and Doolittle 1982).

\section{Proteolysis and TAP Transport}

Proteolysis and transporter associated antigen presentation (TAP) are important for processing of the MHC-I and forming peptide-MHC-I complexes. The cleavage site on the multiepitope peptide vaccine was evaluated by NetChop and MAPPP servers. The number of possible predicted cleavage sites by NetChop and MAPPP servers were 12 and 16, respectively (Fig. 5). The prediction of the TAP binding

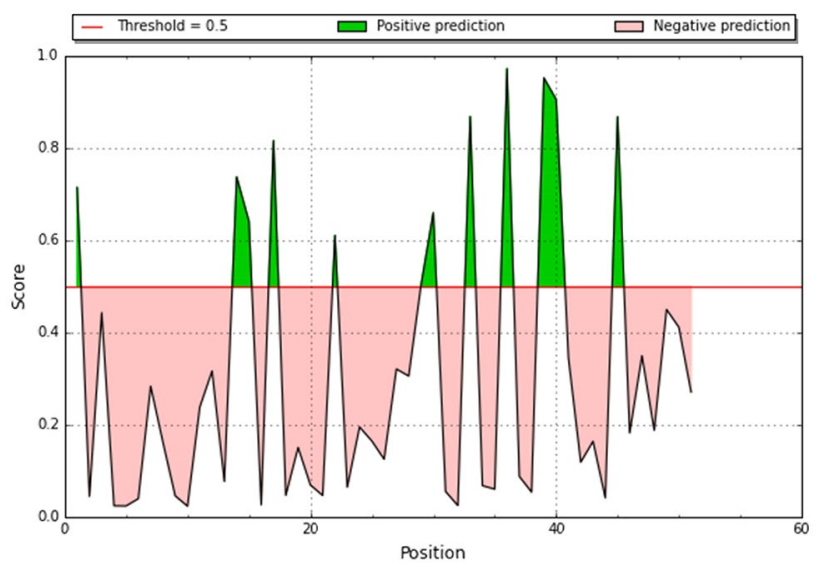

Fig. 5 The predicted proteasome cleavage sites of the multiepitope peptide vaccine. The positive predictions (cleavage sites) are displayed in green while below the threshold value $(0.5)$ predicted regions are in red. (For interpretation of the references to color in this figure legend, the reader is referred to the web version of this article). (Color figure online)

affinity of the multiepitope peptide was achieved using TAPPred server. The results showed three segments with high binding affinity and ten segments with intermediate binding affinity to TAP protein. The high-affinity peptides are illustrated in Table S8.

\section{Determining The 3D Structure of The Multiepitope Peptide Vaccine}

The 3D structure is necessary for determining the conformational B cells epitope by Ellipro server. Therefore, phyre2 and SWISS-MODEL servers were employed to determine the peptide vaccine 3D structure through homology modeling. However, the alignment quality of the query with the template models was only $47 \%$ (low confidence). Due to homology modeling failure, the PEP-FOLD and CABS-fold servers were used for de novo prediction of the 3D structure (Manijeh et al. 2013). The secondary structure is a prerequisite factor for de novo 3D structure prediction.

The obtained 3D structure was refined by GalaxyLoop, ReFOLD, and ModRefiner servers (Fig. 6). The results were submitted to ERRAT and ProSA-web to verify the quality 
A

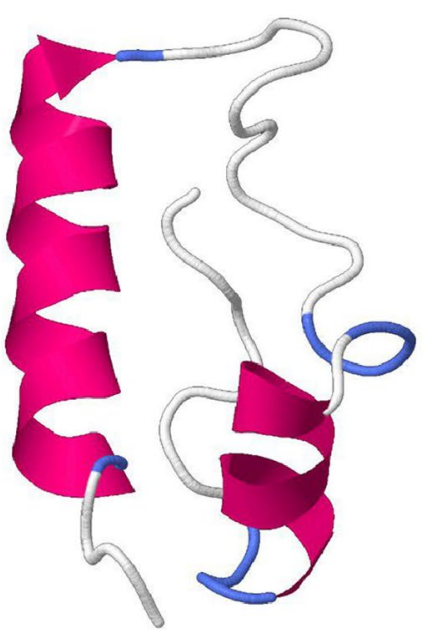

B

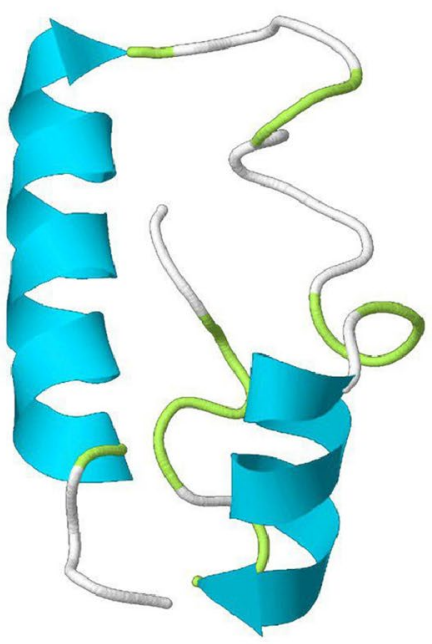

C

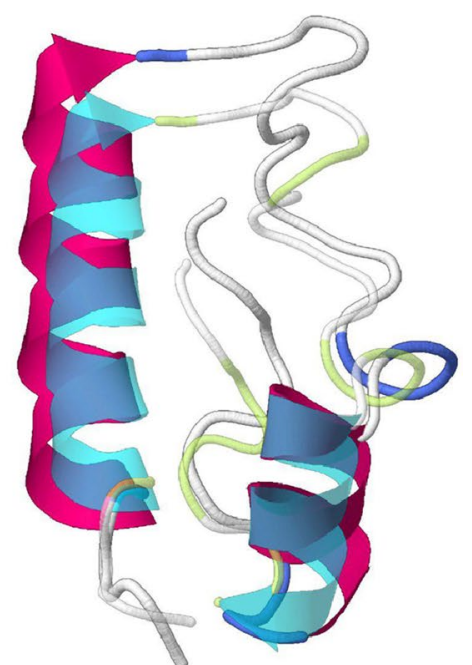

Fig. 6 The predicted tertiary structure of the multiepitope peptide vaccine. a The initial model. b The refined model after precise loop refinement and energy minimization. $\mathbf{c}$ The initial and refined 3D models superimposing

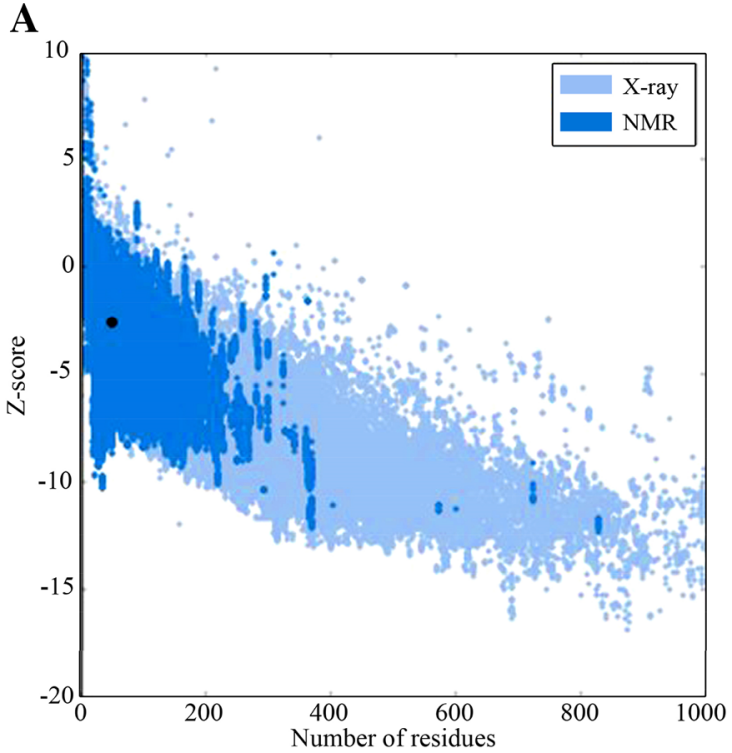

Fig. $7 \mathrm{z}$-Score plot for 3D structure of the construct. The z-score of a the initial model is -2.57 . b The $z$-score of the model after refinement processes is -3.22 . $\mathrm{z}$-Score plot contains z-Scores of all experimentally protein chains in PDB determined by NMR spectroscopy (dark blue) and X-ray crystallography (light blue). The plot shows

and potential errors in a crude 3D model. The best primary model $\mathrm{z}$-score and overall quality factor were -2.57 and 74.41 for ProSA-web and ERRAT, respectively.

The 3D structure refinement was done and the model parameters turned to -3.22 and 100 (Figs. 7, 8).

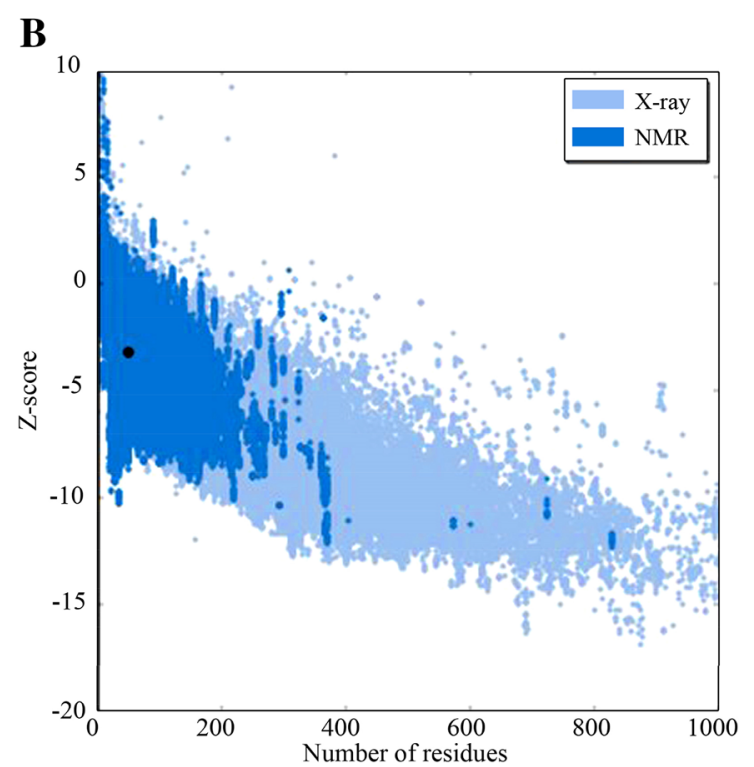

results with a z-Score $\mathrm{r} 10$. The $\mathrm{z}$-Score of the peptide vaccine is presented in large black dot. (For interpretation of the references to color in this figure legend, the reader is referred to the web version of this article). (Color figure online)

For final validation of the refined 3D structures, Ramachandran maps were performed for primary and the refined model by PROCHECK server (Fig. 9). Before refinement, $76 \%$ of residues were located in the favorite regions according to Ramachandran plot and after the refinement, this amount changed to $91 \%$. All related data 

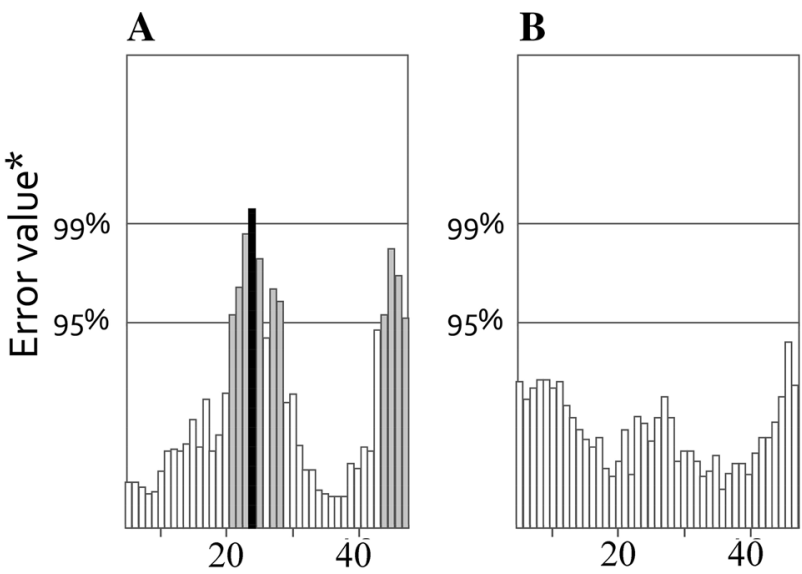

Fig. 8 The overall quality factor plot (ERRAT) of a the initial model is $74.41 \%$ and $\mathbf{b}$ the final model after refinement processes is $100 \%$. In ERRAT plot, regions of the 3D model that can be rejected at the 95\% confidence level are shown in gray lines and regions that can be rejected at the $99 \%$ level are depicted in black lines. The overall quality factor manifests the percentage of protein for which the computed error value falls under the $95 \%$ rejection limit. Good high-resolution structures usually generate values about $95 \%$ or higher of the primary and the refined models are presented in Table S9.

\section{Determining the B Cells Epitopes}

Many studies support the critical role of B cells in antitumor immune response (Tsou et al. 2016). Therefore, the final refined 3D structure was applied as an input in ElliPro and DiscoTope servers for identifying conformational B cells epitopes (Table S10 and Fig. 10). In addition, Identification of the B cell linear epitopes was carried out by BCPRED and ElliPro servers. The multiepitope peptide vaccine had 4 linear and 6 conformational B cells epitopes (Table S11).

\section{Post-translation Modifications}

One of the most determining factors in designing DNA vaccines is post-translational modification of its translated peptide. Post-translational modifications can significantly affect stability and intracellular processing of a peptide for $\mathrm{MHC}$ pathway. The multi-epitope peptide vaccine length was 51 amino acids $(6.35 \mathrm{kDa})$ without any PEST site. The translated peptide does not have any function according to predictions of the utilized servers. All the predicted ubiquitination, phosphorylation, and glycosylation sites are illustrated in

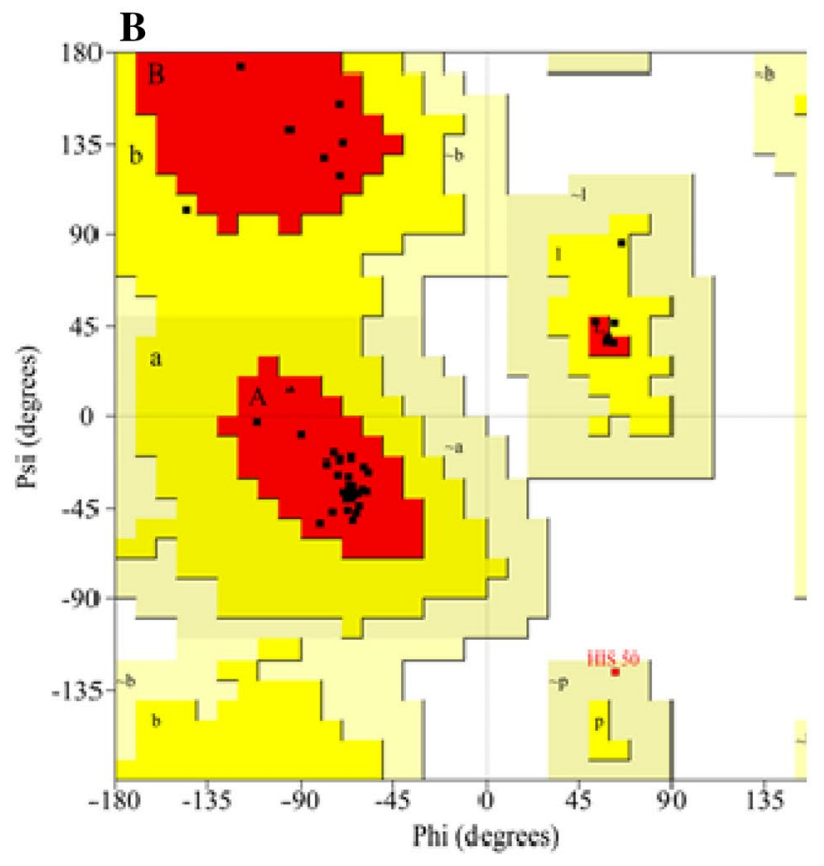

Fig. 9 Validation of the peptide vaccine 3D model using Ramachandran plot. The Ramachandran plots of a the initial model indicated that $76.6 \%$ of residues are located in most-favored regions (a, $\mathrm{b}$ and 1$), 14.9 \%$ in additional allowed regions (a, b, 1, and p), 6.4\% in generously allowed regions $(\sim \mathrm{a}, \sim \mathrm{b}, \sim 1, \sim \mathrm{p})$ and $2.1 \%$ in disallowed regions of the plot and $\mathbf{b}$ the model after refinement revealed that $91.5 \%$ of residues are located in most-favored regions, $6.4 \%$ in additional allowed regions, $2.1 \%$ in generously allowed regions and only $0 \%$ in disallowed regions. Favored and the most-favored regions demonstrated with yellow and red colors, respectively. The generously allowed regions are indicated in pale yellow, and the disallowed regions are in white color. Residues of protein are shown in black squares. (For interpretation of the references to color in this figure legend, the reader is referred to the web version of this article). (Color figure online) 

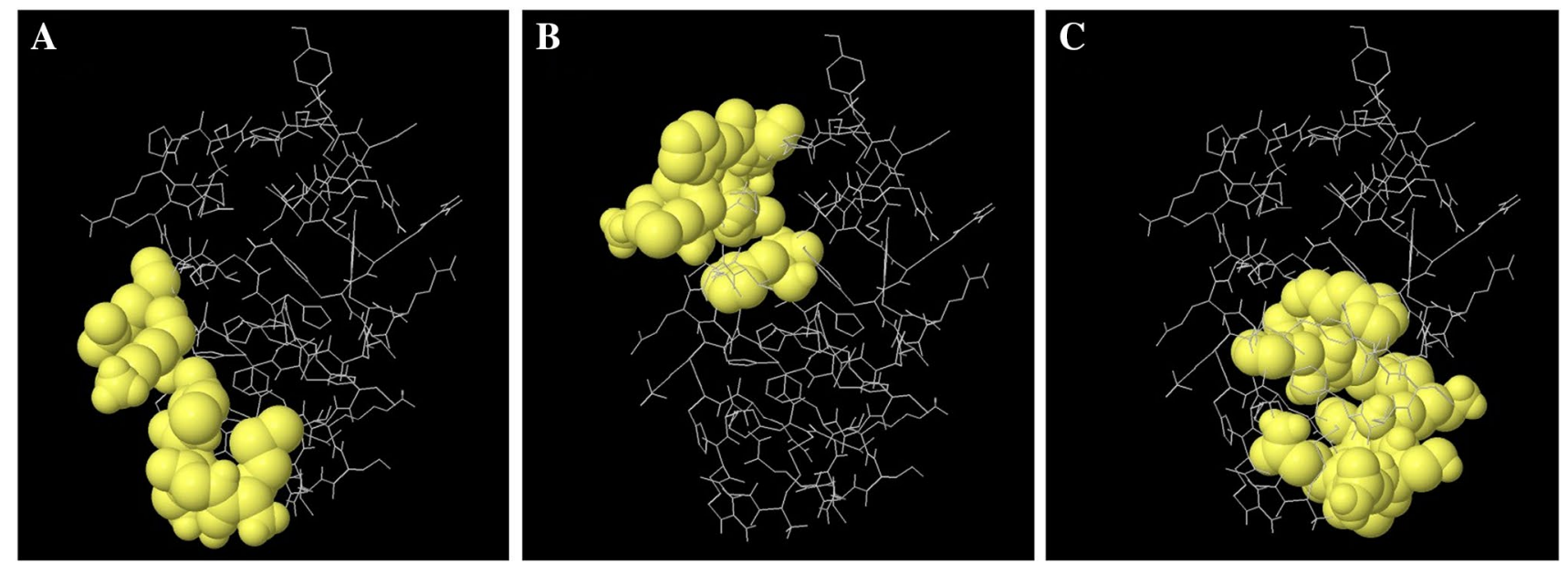

Fig. 10 The predicted conformational B cell epitopes from the 3D structure template are exhibited in a-c ball and stick model. (The residues of predicted epitopes are simulated by yellow balls and white sticks are for non-epitope and core residues). (Color figure online)

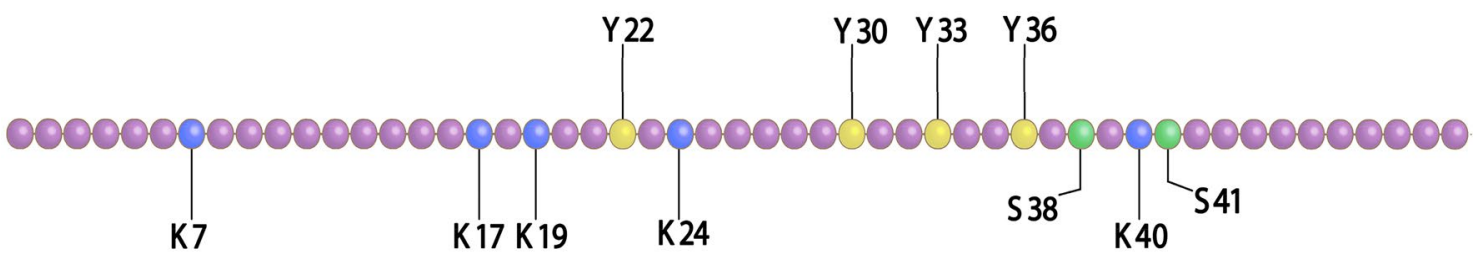

Fig. 11 The predicted ubiquitination and phosphorylation cited at the final construct after post-translational modifications. Ubiquitination and phosphorylation sites are illustrated as blue and yellow beads.

Fig. 11. No N-glycosylation site was predicted for the peptide vaccine. However, two sites of O-glycosylation including the 38 and 41 residues were predicted.

\section{Data Validation}

Due to the importance of the CTLs at cancer immune therapy, intensive CTL epitopes validation was done with different servers. Most of the reassigned CTLs epitopes within the final construct were the same as the epitopes assigned in the first place. As Table S12 illustrates, the peptide vaccine has multiple CTL epitopes with MHC-I binding ability. In addition, the predicted population coverage worldwide for the final construct according to the ability for binding to MHC alleles was $90 \%$ (Table S5).

\section{The Multiepitope DNA Vaccine Development and In Silico Cloning}

Codon adaptation is a way to attain major expression rate for foreign genes. Especially, when the host codon usage differs from that gene originated organism. Therefore, the
The green beads exhibit sites which underwent both phosphorylation and O-glycosylation. $K$ lysine; $S$ serine; and $Y$ tyrosine. (Color figure online)

final construct was reverse translated and codon optimized. The optimized codon adaptation index (CAI) for mouse and bacteria were 0.92 and 0.87 , respectively and the overall GC content was $52.54 \%$ (Fig. 12).

The final construct contained suitable restriction enzymes sites (Fig. 13) and Kozak sequences in its structure. These restriction sites cause ability for cloning into pcDNA3.1 to form a multiepitope DNA vaccine or pET-28a for express at E. coli.

\section{Discussion}

Cancer is one of the leading cause of death in human societies (Stewart 2014). In spite of significant developments in the knowledge about cancer, the current treatments outcomes are not satisfying. Recently, cancer immune therapy has received a lot of attention due to considerable effectiveness and low side effects (Urruticoechea et al. 2010). It has different approaches including cell-mediated immune therapy, monoclonal antibodies, and vaccines (Regan et al. 2016; Lin and Li 2013). 


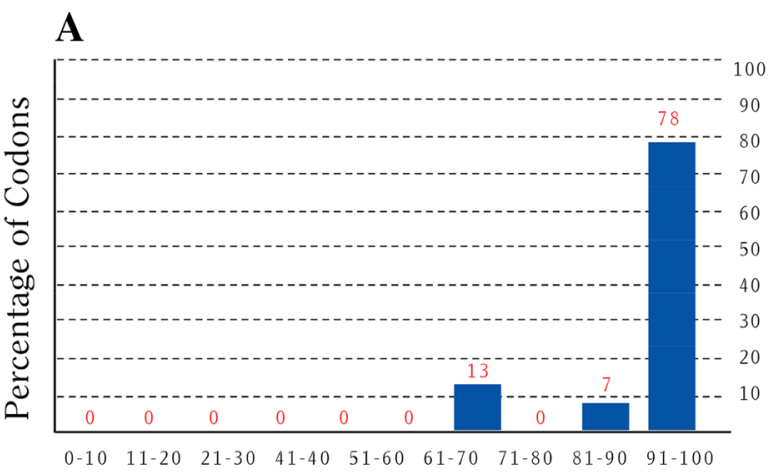

C

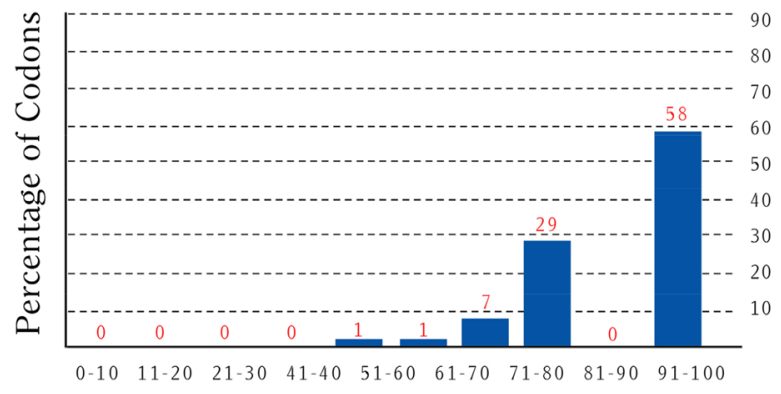

$\mathbf{E}$

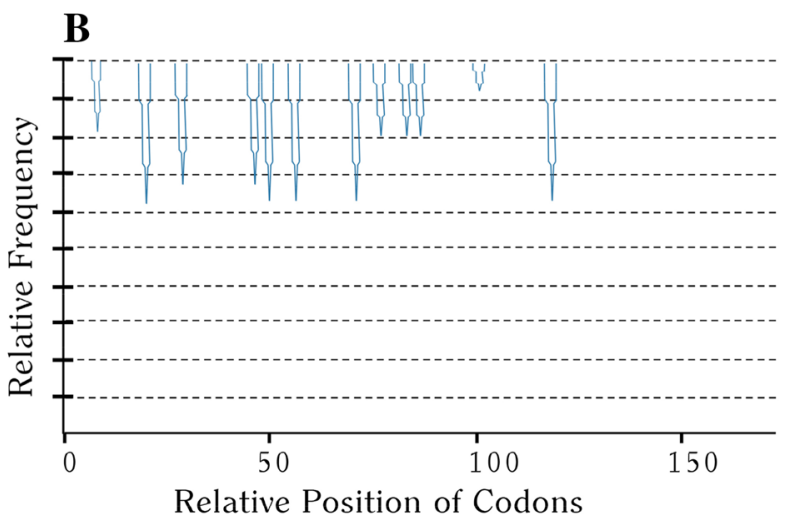

D

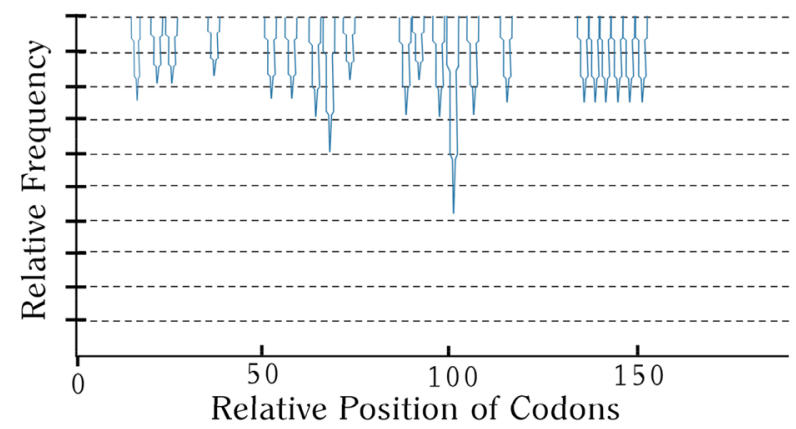

\section{GCTAGCACCATGGGCCGCTGCCAGCACAAAATCGCCGAAATGG TGGCCCTGATGGAAAAACACAAACACCAGTACGACAAAATCCG CGTGCGCCGCTACATCCAGTACCCAAACTACTGCAGCTTCAAAA GCCAGCAGTGCCTGCACCACCACCACCACCACTGACTCGAG}

Fig. 12 The Codon adaptation index value and the codon frequency distribution (CFD) of the DNA sequence. a The CAI value is 0.92 for the murine host. b The codon with frequency distribution of 91-100, $81-90,71-80$ and $61-70$ are respectively $78 \%, 7 \%, 0 \%$ and $13 \%$ in gene sequence for murine host. c The CAI value is 0.87 for $E$. coli. d codon with frequency distribution of 91-100, 81-90, 71-80, 61-70,
51-60 and 41-50 are respectively 58\%, 0\%, 29\%, 7\%, 1\% and $1 \%$ for E. coli host. (The CAI equal 1 and over 0.8 are announced as perfect and good for expression in the chosen host, respectively and codons with values lower than 30 can prevent efficient expression). e The optimized DNA sequence of the final construct

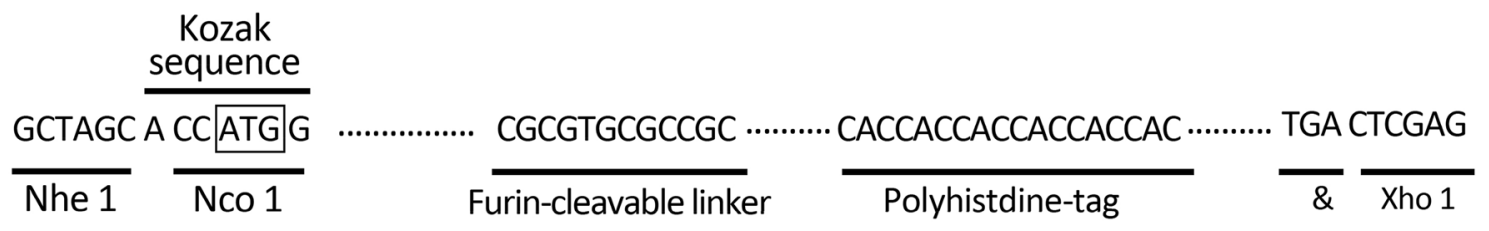

Fig. 13 The schematic diagram of the sequence of the constructed DNA vaccine. It contains Nhe1 and Ncol restriction enzymes sequences at $5^{\prime}$ end and also an Xhol sequence at $3^{\prime}$ ends. A furin- sensetive linker sequence fuses the two epitopes to each other. Also, a Kozak sequence is designed to enhance transcription 
Cancer vaccines have exhibited considerable advantages in clinical trials (Finn 2014). The main purpose of vaccinations therapy is the efficient presentation of appropriate tumor-specific antigens to the patients' immune system. The most suitable antigens for cancer vaccination therapy are TAAs subtypes like CTAs (Vinay et al. 2015; Vigneron 2015). The CTAs expression in normal tissue is extremely rare and malignant transformations cause their overexpression in the cancerous cells (Salmaninejad et al. 2016; Cheng and Mruk 2012). Therefore, CTAs have been introduced as appropriate targets for cancer vaccination and anti-cancer immune response. Many clinical trials based on CTAs vaccines have been completed like MAGE and NYESO antigens vaccine therapy for melanoma patients which had significant outcomes (Fratta et al. 2011).

This is apparent that targeting multiple immunodominant epitopes from different antigens can enhance the vaccination outcome (Buonaguro et al. 2011). Therefore, immunodominant epitopes have long been used to design peptides for vaccination against cancer (Ebert et al. 2009; Iinuma et al. 2014). Immunoinformatic tools and databases are getting more involved in designing novel cancer vaccines. They are one of the main methods for the selection of antigens immunodominant epitopes (Backert and Kohlbacher 2015).

In this study, two CTAs including SYCP1 and ACRBP were utilized for designing a multiepitope DNA and peptide cancer vaccine by bioinformatic tools. SYCP1 and ACRBP expression and up-regulation have been observed in many cancers. Therefore, a peptide vaccine consists of their immunodominant epitopes can exhibit high coverage for a vast variety of cancers. In cancer patients, activation of both cellular and humoral immune response against SYCP1 and ACRBP have been reported which demonstrate immunogenicity of these antigens in the autologous host (Taherian-Esfahani 2016). Cancer vaccines should be assessed by animal experiments before interring clinical trials. This fact is more prominent in cancer which murine tumor models are using vastly (Martin et al. 1986). Therefore, the regions with completely the same amino acid sequences between human and mouse were selected. This can make it possible for applying the animal experiments to human. Selection of epitopes from human segments without considering the murine epitopes may have some disadvantages. A selected immunodominant epitope for human segments may not have the same amino acid residue in comparison with murine. The murine cancer cells may exhibit the antigen with differences in the selected epitope residue. Therefore, it wouldn't be recognized by the immune system which has been provoked against human epitopes. It would be a big sight for an in silico analysis to consider both the mouse and human at the same time for selecting the immunodominant epitopes. This will make the result of in vivo experiments of the designed cancer vaccine more trustable.
In part of cancer vaccination, immune tolerance is a big challenge while utilizing autologous antigens and this problem can be solved by comparison of the antigens sequences with the proteome to select the unique epitopes (Makkouk and Weiner 2015). In this study, the selected antigens regions were compared with both human and mouse proteomes to avoid immune tolerance in both species (Tables S1, S2).

In cellular immune response against cancer, CTLs play the most important role in attacking cancer cells by producing interferon gamma and tumor cells apoptosis induction. Accordingly, CTLs have been shown to associate with better survival for many cancer patients (Kessler and Melief 2007; Chen et al. 2014). Although CTLs have been considered as the main player in anti-tumor response resulting from vaccines, several studies have suggested that the $\mathrm{CD} 4^{+} \mathrm{T}$ cells orchestrate a broad range of immune responses against cancer. This fact is more prominent at peptide vaccination therapy. This cell population differentiates into multiple sublineages which can induce and maintain anti-tumor immune responses (Galaine et al. 2015; Hadrup et al. 2013). Due to the undeniable function of cellular immunity at tumor eradication; at first, the $\mathrm{T}$ cell epitopes which are associated with MHC-I and II binding ability were selected. The two selected regions contained different epitopes for binding to a broad spectrum of HLAs (Tables S3, S4). Restricted binding to specific HLAs could merely be effective in a population with a high frequency of these HLAs (Imanishi 1992). However, restricted binding to specific HLAs limits the coverage to a specific ethnicity because of the dramatic polymorphism of the HLA genes and different expression frequencies in each ethnicity (Khalili et al. 2015). The multiepitope cancer vaccine had about $90 \%$ HLA coverage in worldwide (Table S5) which can be related to containing multiple epitopes from two different CTAs (Bui et al. 2006).

The final construct of multiepitope peptide vaccine was formed by linking the two selected fragments from SYCP1 and ACRBP together with a furin-sensitive linker (Fig. 4). This linker can enhance the CTL role in the immune response. It causes effective processing of the multiepitope cancer vaccine in the trans-Golgi network and endoplasmic reticulum. Therefore, the furin-sensitive linkers are necessary for effective fragments separation (Lu et al. 2004). In addition, the final construct exhibited appropriate immunogenicity for B cells. B cells ability to shape the other immune cells functions by secreting cytokines, providing co-stimulation, and especially presenting antigens has made them important in cancer vaccine therapy. The B cells epitopes improve the peptide vaccine uptake by the B cells, presentation to the $\mathrm{CD} 4$ lymphocytes, and subsequent $\mathrm{CD} 8^{+}$ cells activation (Tsou et al. 2016; Martin and Chan 2006). Therefore, peptide vaccines should contain not only CTL epitopes but also B cells epitopes. The B cells epitopes can be divided into linear and conformational. The multiepitope 
peptide vaccine contained three conformational epitopes in its 3D structure and four linear epitopes (Tables S10, S11).

Many post-translational modifications (PTM) have been introduced for proteins. Some of them like ubiquitination, phosphorylation, and glycosylation can significantly affect the cancer vaccines efficiency. Ubiquitination sites with peptide vaccines facilitate their proper targeting to proteasome, degradation, and epitopes presentation. Therefore, ubiquitination improves the antigen entry into the MHC-I pathway and enhances the $\mathrm{CD} 8^{+} \mathrm{T}$ cell responses (Rodriguez et al. 1998; Leifert et al. 2004). Phosphorylation and glycosylation are two others important PTMs. Phosphorylated antigens could better undergo ubiquitination and proteasomal degradation (Ang and Harper 2005). There are six predicted phosphorylation sites within the multiepitope peptide vaccine which could function as a degradation signal (Zarling et al. 2000; Dougan et al. 2012). The impact of glycosylation on immunogenicity and antigenicity have been mentioned in many studies. Especially, the O-glycosylation sites which cause the epitopes display by both MHC classes (Zarling et al. 2000). PTMs analysis revealed 2 glycosylation sites in the peptide. In general, our peptide vaccine exhibits appropriate PTMs which enhance its immunogenicity and efficacy (Fig. 11).

The multiepitope peptide vaccine has appropriate stability and solubility. Stability and solubility are critical issues in both translation and post-translation. High solubility not only supports stability but also enhances bioavailability, immunogenicity, and decreases undesired side effects (Prasad et al. 2011; Vazquez et al. 2011). It had proteasome cleavage sites and TAP transport affinity which cause epitopes transport to the endoplasmic reticulum to form peptide-MHC complexes. It could provoke CTLs because of its multiple CTL epitopes. The main concern after formation of final construct is to disrupt the initial epitopes of antigens due to manipulations like adding linkers and etc. Therefore, Different servers including SYFPEITHI were employed to validate the final construct according to CTLs binding affinity. The data validation demonstrated that the modifications and manipulations couldn't change the primary epitopes (Table S12). The CTL epitopes were validated by NetCTLpan and NetCTL which integrate MHC-I-binding ability, proteasomal C terminal cleavage, and TAP transport efficiency to identify restricted CTL epitopes.

The main purpose of the final construct was to form multiepitope DNA and peptide vaccines. The final structure was reverse translated and codon optimized for both bacteria and mammalians. Therefore, the final DNA construct would gain the ability for integrating into the pcDNA3.1 vector as DNA vaccine and/or into pET-28a vector for producing the peptide vaccine in bacteria expression system. For the enhancement of the translation process, the Kozak sequence (ACCATG
G) was added to 5' of the DNA construct (Kozak 1987). Its ATG was used to form the start codon (AUG which translates to methionine) in mRNA sequence (Drabkin and RajBhandary 1998). In addition, the GC nucleotides were added to the last nucleotide of the Kozak sequence $(\mathrm{G})$ to form the GGC codon for avoiding from the frameshift (Fig. 10). This codon will be translated to Glycine.

\section{Conclusion}

In this study, a novel peptide vaccine was designed consist of SYCP1 and ACRBP immunodominant CTLs and B cells epitopes for breast cancer immunotherapy. The multiepitope vaccine had about $90 \%$ HLA coverage in worldwide which can be related to containing multiple epitopes from two different CTAs. Therefore, the multiepitope peptide and DNA vaccines efficacy should be evaluated by future in vivo experimental studies.

Acknowledgements This work is a part of the Ph.D. thesis and financially supported by the Science and Research Branch of Islamic Azad University, Tehran, Iran.

\section{Compliance with Ethical Standards}

Conflict of interest The authors declare that they have no competing interests.

\section{References}

Ang XL, Harper JW (2005) SCF-mediated protein degradation and cell cycle control. Oncogene 24(17):2860

Arpino G, Milano M, De Placido S (2015) Features of aggressive breast cancer. Breast 24(5):594-600

Backert L, Kohlbacher O (2015) Immunoinformatics and epitope prediction in the age of genomic medicine. Genome Med 7(1):119

Beatty GL, Gladney WL (2015) Immune escape mechanisms as a guide for cancer immunotherapy. Clin Cancer Res 21(4):687-692

Bhasin M, Raghava G (2004) Prediction of CTL epitopes using QM, SVM and ANN techniques. Vaccine 22(23):3195-3204

Blaszczyk M et al (2013) CABS-fold: server for the de novo and consensus-based prediction of protein structure. Nucleic Acids Res 41(W1):W406-W411

Blom N, Gammeltoft S, Brunak S (1999) Sequence and structure-based prediction of eukaryotic protein phosphorylation sites 1. J Mol Biol 294(5):1351-1362

Blom N et al (2004) Prediction of post-translational glycosylation and phosphorylation of proteins from the amino acid sequence. Proteomics 4(6): 1633-1649

Bui H-H et al (2006) Predicting population coverage of T-cell epitopebased diagnostics and vaccines. BMC Bioinform 7(1):153

Buonaguro L et al (2011) Translating tumor antigens into cancer vaccines. Clin Vaccine Immunol 18(1):23-34

Chen P, Rayner S, Hu K-h (2011) Advances of bioinformatics tools applied in virus epitopes prediction. Virol Sin 26(1):1-7 
Chen $\mathrm{Z}$ et al (2014) Intratumoral $C D 8^{+}$cytotoxic lymphocyte is a favorable prognostic marker in node-negative breast cancer. PLoS ONE 9(4):e95475

Cheng CY, Mruk DD (2012) The blood-testis barrier and its implications for male contraception. Pharmacol Rev 64(1):16-64

Chomez P et al (2001) An overview of the MAGE gene family with the identification of all human members of the family. Can Res 61(14):5544-5551

Colovos C, Yeates TO (1993) Verification of protein structures: patterns of nonbonded atomic interactions. Protein Sci 2(9):1511-1519

Costa $\mathrm{Y}$ et al (2005) Two novel proteins recruited by synaptonemal complex protein 1 (SYCP1) are at the centre of meiosis. J Cell Sci 118(12):2755-2762

Coulie PG et al (1994) A new gene coding for a differentiation antigen recognized by autologous cytolytic $\mathrm{T}$ lymphocytes on HLA-A2 melanomas. J Exp Med 180(1):35-42

de L Peres et al (2015) Peptide vaccines in breast cancer: the immunological basis for clinical response. Biotechnol Adv 33(8):1868-1877

Demetris Iacovides SM, Achilleos C, Strati K (2013) Shared mechanisms in stemness and carcinogenesis: lessons from oncogenic viruses. Front Cell Infect Microbiol 3:66

Dougan D, Micevski D, Truscott K (2012) The N-end rule pathway: from recognition by $\mathrm{N}$-recognins, to destruction by AAA + proteases. Biochim Biophys Acta 1823(1):83-91

Doytchinova IA, Guan P, Flower DR (2006) EpiJen: a server for multistep T cell epitope prediction. BMC Bioinform 7(1):131

Drabkin HJ, RajBhandary UL (1998) Initiation of protein synthesis in mammalian cells with codons other than AUG and amino acids other than methionine. Mol Cell Biol 18(9):5140-5147

Ebert LM et al (2009) A long, naturally presented immunodominant epitope from NY-ESO-1 tumor antigen: implications for cancer vaccine design. Can Res 69(3):1046-1054

El-Manzalawy Y, Dobbs D, Honavar V (2008) Predicting linear B-cell epitopes using string kernels. J Mol Recognit 21(4):243-255

Erenpreisa J, Cragg MS (2013) Three steps to the immortality of cancer cells: senescence, polyploidy and self-renewal. Cancer Cell Int 13(1):92

Finn OJ (2003) Cancer vaccines: between the idea and the reality. Nat Rev Immunol 3(8):630

Finn OJ (2014) Vaccines for cancer prevention: a practical and feasible approach to the cancer epidemic. Cancer Immunol Res 2(8):708-713

Fratta E et al (2011) The biology of cancer testis antigens: putative function, regulation and therapeutic potential. Mol Oncol 5(2):164-182

Galaine J et al (2015) Interest of tumor-specific CD4 T helper 1 cells for therapeutic anticancer vaccine. Vaccines 3(3):490-502

Gasteiger E et al (2005) Protein identification and analysis tools on the ExPASy server. Springer, Berlin

Ghahremani F et al (2018) AS1411 aptamer conjugated gold nanoclusters as a targeted radiosensitizer for megavoltage radiation therapy of 4T1 breast cancer cells. RSC Adv 8(8):4249-4258

Gjerstorff MF, Andersen MH, Ditzel HJ (2015) Oncogenic cancer/ testis antigens: prime candidates for immunotherapy. Oncotarget 6(18): 15772

Goodwin D, Simerska P, Toth I (2012) Peptides as therapeutics with enhanced bioactivity. Curr Med Chem 19(26):4451-4461

Greenbaum J et al (2011) Functional classification of class II human leukocyte antigen (HLA) molecules reveals seven different supertypes and a surprising degree of repertoire sharing across supertypes. Immunogenetics 63(6):325-335

$\mathrm{Gu}$ Y et al (2017) Vaccination with a paramyosin-based multiepitope vaccine elicits significant protective immunity against trichinella spiralis infection in mice. Front Microbiol 8:1475
Guan P et al (2003) MHCPred: a server for quantitative prediction of peptide-MHC binding. Nucleic Acids Res 31(13):3621-3624

Guex N, Peitsch MC, Schwede T (2009) Automated comparative protein structure modeling with SWISS-MODEL and Swiss-PdbViewer: a historical perspective. Electrophoresis 30(S1):S162-S173

Hadrup S, Donia M, Straten P (2013) Effector CD4 and CD8 T cells and their role in the tumor microenvironment. Cancer Microenviron 6(2):123-133

Iinuma $\mathrm{H}$ et al (2014) Phase I clinical study of multiple epitope peptide vaccine combined with chemoradiation therapy in esophageal cancer patients. J Transl Med 12(1):84

Ikai A (1980) Thermostability and aliphatic index of globular proteins. J Biochem 88(6): 1895-1898

Imanishi T (1992) Allete and haplotype frequencies for HLA and complement loci in various ethnic groups. In: Tsuji K, Aizawa M, Sasazuki T (eds) HLA 1991. Oxford University Press, Oxford, pp 1065-1220

Jensen KK et al (2018) Improved methods for predicting peptide binding affinity to MHC class II molecules. Immunology 154:394-406

Karkada M et al (2013) Tumor inhibition by DepoVax-based cancer vaccine is accompanied by reduced regulatory/suppressor cell proliferation and tumor infiltration. ISRN Oncol 2013:75321

Kelley LA, Sternberg MJ (2009) Protein structure prediction on the Web: a case study using the Phyre server. Nat Protoc 4(3):363-371

Kessler J, Melief C (2007) Identification of T-cell epitopes for cancer immunotherapy. Leukemia 21(9):1859

Khalili S et al (2015) In silico analyses of Wilms' tumor protein to designing a novel multi-epitope DNA vaccine against cancer. J Theor Biol 379:66-78

Khanfir A et al (2013) Prognostic factors and survival in metastatic breast cancer: a single institution experience. Rep Pract Oncol Radiother 18(3):127-132

Ko J et al (2012) GalaxyWEB server for protein structure prediction and refinement. Nucleic Acids Res 40(W1):W294-W297

Kozak M (1987) An analysis of 5'-noncoding sequences from 699 vertebrate messenger RNAs. Nucleic Acids Res 15(20):8125-8148

Kozakov D et al. (2010) Achieving reliability and high accuracy in automated protein docking: ClusPro, PIPER, SDU, and stability analysis in CAPRI rounds 13-19. Proteins Struct Funct Bioinform 78(15):3124-3130

Kraus MH et al (1987) Overexpression of the EGF receptor-related proto-oncogene erbB-2 in human mammary tumor cell lines by different molecular mechanisms. EMBO J 6(3):605

Kringelum JV et al (2012) Reliable B cell epitope predictions: impacts of method development and improved benchmarking. PLoS Comput Biol 8(12):e1002829

Kubuschok B et al (2004) Expression of cancer testis antigens in pancreatic carcinoma cell lines, pancreatic adenocarcinoma and chronic pancreatitis. Int J Cancer 109(4):568-575

Kyte J, Doolittle RF (1982) A simple method for displaying the hydropathic character of a protein. J Mol Biol 157(1):105-132

Larsen MV et al (2007) Large-scale validation of methods for cytotoxic T-lymphocyte epitope prediction. BMC Bioinform 8(1):424

Laskowski RA et al (1993) PROCHECK: a program to check the stereochemical quality of protein structures. J Appl Crystallogr 26(2):283-291

Leifert JA et al (2004) Targeting plasmid-encoded proteins to the antigen presentation pathways. Immunol Rev 199(1):40-53

Lin C, Li Y (2013) The role of peptide and DNA vaccines in myeloid leukemia immunotherapy. Cancer Cell Int 13(1):13 
Lu J et al (2004) Multiepitope Trojan antigen peptide vaccines for the induction of antitumor CTL and Th immune responses. J Immunol 172(7):4575-4582

Luo G et al (2002) Expression of cancer-testis genes in human hepatocellular carcinomas. Cancer Immun Arch 2(1):11

Lurquin C et al (1997) Two members of the HumanMAGEBGene family located in Xp21. 3 are expressed in tumors of various histological origins. Genomics 46(3):397-408

Magnan CN, Randall A, Baldi P (2009) SOLpro: accurate sequencebased prediction of protein solubility. Bioinformatics 25(17):2200-2207

Mahdavi M et al (2012) Linear and conformational B cell epitope prediction of the HER 2 ECD-subdomain III by in silico methods. Asian Pac J Cancer Prev 13(7):3053-3059

Makkouk A, Weiner GJ (2015) Cancer immunotherapy and breaking immune tolerance: new approaches to an old challenge. Can Res 75(1):5-10

Manijeh M et al (2013) In silico design of discontinuous peptides representative of B and T-cell epitopes from HER2-ECD as potential novel cancer peptide vaccines. Asian Pac J Cancer Prev 14(10):5973-5981

Martin F, Chan AC (2006) B cell immunobiology in disease: evolving concepts from the clinic. Annu Rev Immunol 24:467-496

Martin DS et al (1986) Role of murine tumor models in cancer treatment research. Can Res 46(4 Part 2):2189-2192

Mashino K et al (2001) Expression of multiple cancer-testis antigen genes in gastrointestinal and breast carcinomas. Br J Cancer 85(5):713

Maupetit J, Derreumaux P, Tuffery P (2009) PEP-FOLD: an online resource for de novo peptide structure prediction. Nucleic Acids Res 37(suppl_2):W498-W503

McGuffin LJ et al (2017) Accurate template based modelling in CASP12 using the IntFOLD4-TS, ModFOLD6 and ReFOLD methods. Proteins 86:335-344

Moradi J et al (2017) Designing a novel multi-epitope DNA-based vaccine against tuberculosis: in silico approach. Jundishapur J Microbiol 10(3):e43950

Nezafat $\mathrm{N}$ et al (2014) A novel multi-epitope peptide vaccine against cancer: an in silico approach. J Theor Biol 349:121-134

Nielsen AY, Gjerstorff MF (2016) Ectopic expression of testis germ cell proteins in cancer and its potential role in genomic instability. Int J Mol Sci 17(6):890

Norell H et al (2010) Vaccination with a plasmid DNA encoding HER-2/neu together with low doses of GM-CSF and IL-2 in patients with metastatic breast carcinoma: a pilot clinical trial. J Transl Med 8(1):53

Ono $\mathrm{T}$ et al (2001) Identification of proacrosin binding protein sp32 precursor as a human cancer/testis antigen. Proc Natl Acad Sci 98(6):3282-3287

Pandey RK, Bhatt TK, Prajapati VK (2018) Novel immunoinformatics approaches to design multi-epitope subunit vaccine for malaria by investigating anopheles salivary protein. Sci Rep 8(1):1125

Patronov A, Doytchinova I (2013) T-cell epitope vaccine design by immunoinformatics. Open Biol 3(1):120139

Ponomarenko J et al (2008) ElliPro: a new structure-based tool for the prediction of antibody epitopes. BMC Bioinform 9(1):514

Prasad S, Khadatare PB, Roy I (2011) Effect of chemical chaperones in improving the solubility of recombinant proteins in Escherichia coli. Appl Environ Microbiol 77(13):4603-4609

Radivojac P et al (2010) Identification, analysis, and prediction of protein ubiquitination sites. Proteins 78(2):365-380

Rammensee H-G et al (1999) SYFPEITHI: database for MHC ligands and peptide motifs. Immunogenetics 50(3-4):213-219
Reche PA et al (2004) Enhancement to the RANKPEP resource for the prediction of peptide binding to MHC molecules using profiles. Immunogenetics 56(6):405-419

Redig AJ, McAllister SS (2013) Breast cancer as a systemic disease: a view of metastasis. J Intern Med 274(2):113-126

Regan D et al (2016) Cancer immunotherapy in veterinary medicine: current options and new developments. Vet J 207:20-28

Robbins PF et al (1996) A mutated beta-catenin gene encodes a melanoma-specific antigen recognized by tumor infiltrating lymphocytes. J Exp Med 183(3):1185-1192

Rodriguez F et al (1998) DNA immunization with minigenes: low frequency of memory cytotoxic $\mathrm{T}$ lymphocytes and inefficient antiviral protection are rectified by ubiquitination. J Virol 72(6):5174-5181

Sahin U et al (2000) Expression of cancer testis genes in human brain tumors. Clin Cancer Res 6(10):3916-3922

Salmaninejad A et al (2016) Cancer/testis antigens: expression, regulation, tumor invasion, and use in immunotherapy of cancers. Immunol Invest 45(7):619-640

Sankar S et al (2017) Short peptide epitope design from hantaviruses causing HFRS. Bioinformation 13(7):231

Schumacher TN, Schreiber RD (2015) Neoantigens in cancer immunotherapy. Science 348(6230):69-74

Shastri N, Schwab S, Serwold T (2002) Producing nature's gene-chips: the generation of peptides for display by MHC class I molecules. Annu Rev Immunol 20(1):463-493

Shin W-H et al (2014) Prediction of protein structure and interaction by GALAXY protein modeling programs. Bio Design 2(1):1-11

Shuid AN, Kempster R, McGuffin LJ (2017) ReFOLD: a server for the refinement of 3D protein models guided by accurate quality estimates. Nucleic Acids Res. https://doi.org/10.1093/nar/gkx249

Singh H, Raghava G (2003) ProPred1: prediction of promiscuous MHC Class-I binding sites. Bioinformatics 19(8):1009-1014

Soria-Guerra RE et al (2015) An overview of bioinformatics tools for epitope prediction: implications on vaccine development. $\mathrm{J}$ Biomed Inform 53:405-414

Steentoft C et al (2013) Precision mapping of the human O-GalNAc glycoproteome through SimpleCell technology. EMBO J 32(10): 1478-1488

Stewart BW, Christopher P (2014) Wild World cancer report. WHO, Geneva. ISBN 978-92-832-0429-9

Stranzl T et al (2010) NetCTLpan: pan-specific MHC class I pathway epitope predictions. Immunogenetics 62(6):357-368

Subramanian N, Chinnappan S (2013) Prediction of promiscuous epitopes in the e6 protein of three high risk human papilloma viruses: a computational approach. Asian Pac J Cancer Prev 14(7):4167-4175

Taherian-Esfahani $\mathrm{Z}$ et al (2016) Cancer-testis antigens: a novel group of tumor biomarkers in ovarian cancers. Iran J Cancer Prev 9(6): 4993

Tammela $\mathrm{J}$ et al (2006) OY-TES-1 expression and serum immunoreactivity in epithelial ovarian cancer. Int J Oncol 29(4):903-910

Tang C-T et al (2015) An epitope-substituted DNA vaccine improves safety and immunogenicity against dengue virus type 2 . PLoS Negl Trop Dis 9(7):e0003903

Tarnowski M et al (2016) Expression of cancer testis antigens in colorectal cancer: new prognostic and therapeutic implications. Dis Mark. https://doi.org/10.1155/2016/1987505

Thundimadathil J (2012) Cancer treatment using peptides: current therapies and future prospects. J Amino Acids. https://doi. org/10.1155/2012/967347

Tiptiri-Kourpeti A et al (2016) DNA vaccines to attack cancer: strategies for improving immunogenicity and efficacy. Pharmacol Ther 165:32-49 
Tiriveedhi V et al (2013) Mammaglobin-A cDNA vaccination of breast cancer patients induces antigen-specific cytotoxic $\mathrm{CD} 4^{+}$ICOS hi T cells. Breast Cancer Res Treat 138(1):109-118

Tsou $\mathrm{P}$ et al (2016) The emerging role of B cells in tumor immunity. Can Res 76(19):5597-5601

Türeci Ö et al (1998) Identification of a meiosis-specific protein as a member of the class of cancer/testis antigens. Proc Natl Acad Sci 95(9):5211-5216

Urruticoechea A et al (2010) Recent advances in cancer therapy: an overview. Curr Pharm Des 16(1):3-10

Vazquez E, Corchero JL, Villaverde A (2011) Post-production protein stability: trouble beyond the cell factory. Microb Cell Fact 10(1):60

Vigneron N (2015) Human tumor antigens and cancer immunotherapy. BioMed Res Int. https://doi.org/10.1155/2015/948501

Vinay DS et al (2015) Immune evasion in cancer: Mechanistic basis and therapeutic strategies. Semin Cancer Biol 35:S185-S198

Vita $\mathrm{R}$ et al (2009) The immune epitope database 2.0. Nucleic Acids Res 38(suppl_1):D854-D862

Weiskopf D et al (2013) Comprehensive analysis of dengue virus-specific responses supports an HLA-linked protective role for $\mathrm{CD} 8^{+}$ T cells. Proc Natl Acad Sci 110(22):E2046-E2053

Whitehurst AW et al (2010a) Tumor antigen acrosin binding protein normalizes mitotic spindle function to promote cancer cell proliferation. Cancer Res 70:7652-7661
Whitehurst AW et al (2010b) Tumor antigen acrosin binding protein normalizes mitotic spindle function to promote cancer cell proliferation. Can Res 70(19):7652-7661

Whiteside TL et al (2011) Mechanisms of tumor escape from the immune system: adenosine-producing Treg, exosomes and tumor-associated TLRs. Bull Cancer 98(2):E25-E31

Wiederstein M, Sippl MJ (2007) ProSA-web: interactive web service for the recognition of errors in three-dimensional structures of proteins. Nucleic Acids Res 35(suppl_2):W407-W410

Xia K et al (2014) Common genetic variants on 1p13. 2 associate with risk of autism. Mol Psychiatry 19(11):1212

$\mathrm{Xu} \mathrm{D}$, Zhang Y (2011) Improving the physical realism and structural accuracy of protein models by a two-step atomic-level energy minimization. Biophys J 101(10):2525-2534

Yang B et al (2014) DNA vaccine for cancer immunotherapy. Hum Vaccines Immunother 10(11):3153-3164

Zajac P et al (2017) MAGE-A antigens and cancer immunotherapy. Front Med 4:18

Zarling AL et al (2000) Phosphorylated peptides are naturally processed and presented by major histocompatibility complex class I molecules in vivo. J Exp Med 192(12):1755-1762 